\title{
Escape from conflict: I. The effects of increasing difficulty of discrimination'
}

THOMAS J. TIGHE AND ROBERT N. LEATON ${ }^{2}$

Rats were trained in successive brightness discriminations with access to a lever which, when pressed, temporarily turned out the lights constituting $S_{+}$and $S_{-}$. There were no progressive changes in the lever pressing of a group which received a series of discriminations of gradually increased difficulty, but there was a significant increase in the lever activity of a group which was suddenly confronted with a difficult problem following prolonged training on an easy problem.

Miller (1959) has suggested that conflict may induce drives over and above the original ones motivating the conflict behavior and that responses which remove $S$ from the conflict situation may be reinforced by the reduction of such drives. However, there has been no unambiguous demonstration of escape from conflict. Although Egger (1960) found that rats learned to press a bar which terminated a stimulus associated with both food and shock, their rate of pressing was comparable to that of control Ss for whom pressing produced only stimulus change. Employing the same food-shock conflict-building technique, Hearst \& Sidman (1961) found that only four of eight Ss reliably terminated the concurrently positive and negative stimulus and their results were confounded by the lack of controls for several potentially significant variables including $\mathrm{S}^{\mathrm{D}}$ aversion, stimulus change, and satiation. Moreover, procedures which employ electric shock to generate the withdrawal component of conflict behavior raise the possibility that behavior which is considered to define escape from conflict is in fact escape from conditioned fear. The present experiment sought to obviate this difficulty by employing a conflict-building technique which did not use noxious stimulation.

\section{Method}

The Ss were four male and six female naive albino rats of the Sprague-Dawley strain approximately 180 days old. They were maintained at 80 to $85 \%$ of their ad lib feeding weight and run $24 \mathrm{hr}$. hungry. The Ss received brightness discrimination training in a straight double-alley maze $45 \mathrm{in}$. long, 8-1/2 in. wide, and 9 in. deep, and painted flat gray throughout. A clear Plexiglas, guillotine door separated the 12 in. long start box from two 4 in. wide alleys beginning $5 \mathrm{in}$. in front of the start box door. The top of each alley consisted of frosted Plexiglas running from the start box door to the end of the alley. Each alley was independently illuminated by a row of sixteen $7-1 / 2$ w bulbs wired in series with a variable transformer and mounted in a wooden box above the Plexiglas alley ceiling which formed the bottom of the box.

A U-shaped lever made of 1/8-in. steel rod was mounted 2 in. above the floor in the rear wall of the start box. The lever was 4 in. wide and protruded 2 in. from the wall of the start box. Lever presses shut off the lights in both alleys for $7 \mathrm{sec}$. Both total lever presses and light terminations were counted. The apparatus was housed in a small room illuminated solely by a 7 w light which provided virtually no illumination to the interior of the maze.
All Ss received three days of pretraining to run and to eat in the maze. During this period the lever was removed from the start box and the brightness of each alley was $5.13 \mathrm{ft}$. $-\mathrm{L}$. Throughout discrimination training $S s$ received ten daily, massed, noncorrection trials. On each trial $S$ was detained in the start box for $1 \mathrm{~min}$. before the transparent door to the alleys was opened. During this period the discriminanda were clearly visible to $S$, and $S$ had ready access to the lever which, when pressed, shut off the discriminanda for $7 \mathrm{sec}$. Reinforcement consisted of a $94-\mathrm{mg}$ pellet. Five Ss (Group I) were given brightness discriminations of progressively increasing difficulty over a period of 70 days. The initial problem consisted of a $19.6 \mathrm{vs} .0 .46 \mathrm{ft} .-\mathrm{L}$. discrimination. In successive discriminations the $.46 \mathrm{ft}-\mathrm{L}$. value remained constant for all $\mathrm{Ss}$ while the brighter illumination was decreased to the following thirteen values ( in ft.-L. measured at the beginning of the alley by an S.E.I. photometer): $14.50,9.20,5.13,4.60,2.71,1.88,1.54,1.30,1.06, .94, .75, .62, .57$. Ss were run in each discrimination until 9 correct choices were made on each of two successive days.

In Group II, each of five Ss was matched to a Group I S on the basis of speed of learning the initial problem. Group II Ss continued to receive training in the initial (easy) problem but reinforcement was matched on a trial by trial basis to the reinforcements received by his paired Group I S. After 70 days of such training Group II Ss were switched to the .75 vs. $.46 \mathrm{ft}$. $-\mathrm{L}$. discrimination and given 13 days of training with $100 \%$ reinforcement for correct responses. This switch in illumination was toward S+ for three Ss and toward S- for two Ss.

Within both experimental treatments "bright" and "dim" were used equally often as the positive and negative stimuli. The frequency of lever presses (both light-out and total presses) were recorded on each trial throughout training.

\section{Resulis}

Each Group I S reached a problem which was apparently insoluble. Two Ss attained Problem 14 (.57 vs. $.46 \mathrm{ft} .-\mathrm{L}$.$) ; one of these Ss received 270$ training trials without approaching criterion while the other $S$ received 210 trials. The remaining Ss progressed to Problems 13,12 , and 11 , and received 220,220 , and 330 training trials, respectively. Group II Ss maintained close to $100 \%$ correct performance after reaching criterion on Problem 1.

In view of the individual differences in learning rates, lever pressing behavior was analyzed in relation to tenths of the total training period. Despite the marked decline in correct responses by Group I Ss during their terminal problems, and the corresponding reduction in the number of reinforcements for Group II Ss, neither group showed systematic changes in lever pressing during the 70 days of testing and no differences between the groups were found (all $\mathrm{F}$ values $<1$ ). Also, $t$ tests comparing lever pressing for criterion and noncriterion trials did not approach significance (all $t$ values $<1$ ). Over the 70 days of testing the mean number of daily light terminations was 13.8 and 13.6 for Groups I and II, respectively. The corresponding mean number of total lever presses was 24.2 and 21.6. These means remained relatively stable from day to day. 


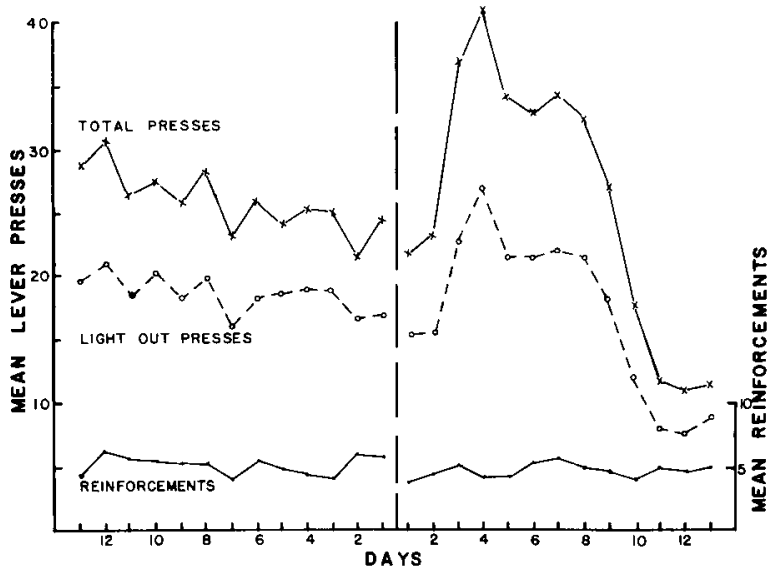

Fig. 1. Lever pressing behavior of Group II during the last 13 days before the introduction of the difficult discrimination and during the 13 post-change days.

In contrast, when suddenly changed to their difficult problem, Group II Ss showed a marked increase in lever pressing (see Fig. 1). A trials by Ss analysis of variance for the 13 post-change days yielded $F=22.1$ $(p<.001, \mathrm{df}=12 / 48)$ for total presses and $F=19.5$ $(p<.001, d f=12 / 48)$ for light-out presses. The same analysis for the 13 pre-change days yielded $F<1$ for both response measures. When the analysis of postchange trials was limited to the first four days an $\mathbf{F}=25.9(\mathrm{p}<.001, \mathrm{df}=3 / 12)$ was found for total presses and an $F=39.2(p<.001$, df = 3/12) was found for lightout presses. Thus, the increase in lever presses, considered alone, was statistically significant. The fall in the curves from day 4 through day 13 was also statistically significant $(F=42.4$ and 31.2 for total presses and light-out presses, respectively, $\mathrm{p}<.001$, df $=9 / 36$ ) .

The mean daily correct responses for these Ss for the 13 pre-change days and the 13 post-change days was 9.7 and 4.6, respectively. No S was approaching criterion on the difficult problem at the end of the 13 days of testing and there were no systematic changes in correct choice performance over the 13 days. It should be noted that the number of reinforcements received by these Ss did not vary significantly with the increase in problem difficulty even though $100 \%$ reinforcement for correct responses was introduced at this point (see Fig.1). The mean daily number of pellets received during the 13 days immediately preceding and following the change in problem difficulty was 5.1 and 4.6 , respectively $(t=$ $1.31, \mathrm{p}>.20)$.

Discussion

The increased lever pressing of Group II when suddenly faced with a difficult discrimination is consistent with the assumption that conflict produced by competing, incompatible response tendencies induces a drive state which can motivate responses which remove cues to that conflict. The (approximately) 20 trial lag between the introduction of the difficult discrimination and the marked increase in lever pressing may reflect the need for a number of training trials with the new problem to generate a strong state of conflict and to condition it to cues associated with introduction of $S$ to the start box. The eventual decline in lever pressing in Group II can be accounted for if it is assumed that adaptation processes ultimately reduced the motivational effectiveness of conflict below the level necessary to produce escape. Adaptation processes may also underlie the failure to find increased lever pressing in Group I. Since in the latter case the conflict was increased in small increments, adaptation may have kept the effective conflict at any given time from reaching the level necessary to produce escape.

The assumption that Group II Ss exhibited escape from conflict should be examined in the light of several aspects of procedure. Motivation of the increased lever pressing by unconditioned aversive properties of S+ and $S-$ seems ruled out by the fact that the problem change was toward dimmer illumination. Also, since lever responses after the start box door was opened were almost nonexistent both before and after problem change, the increase was not due to the Ss simply staying longer in the vicinity of the lever. In view of the delayed nature of the change in lever activity, it seems unlikely that stimulus change per se was a factor in the change, but the present design does not rule out this possibility or the possibility that conflict may have had a potentiating effect on general activity or on lever pressing for stimulus change. The contribution of such processes must be assessed in future experiments.

\section{References}

Egger, M. D. Will rats work to escape from sonflict? Paper presented at the meetings of the American Psychological Association, 1960 .

Hearst, E., \& Sidman, M. Some behavioral effects of a concurrently positive and negative stimulus. J. exp. Anal. Behav., 1961, 4, 251-256.

Miller, N. E Liberalization of basic S-R concepts: extensions to conflict behavior, motivation, and social learning. In $\mathrm{S}$. Koch (Ed.), Psychology: a study of a science. Vol. 2. New York: McGraw-Hill, 1959. Pp. 196-292.

\section{Notes}

1. Supported in part by grant GB 1673 from the National Science Foundation and by Public Health Research Grant HD01850-01 from the National Institute of Child Health and Human Development. 2. The research has been fully collaborative and the order of authorship, having been determined by chance, coes not indicate priority of any sort. 